\title{
Hematological Changes in Threatened Preterm Labor (Hemoglobin \& Platelets)
}

A.M.ALGazar, AW.Mourad , M.A.Mahmoud, and N.M.Mohamed

Obstetrics,Gynecology Dept., Faculty of Medicine, Benha Univ., Benha, Egypt

E-Mail:Nesma@gmail.com

\begin{abstract}
In Threatened PTL patients, due to inflammation and possible ongoing ischemia process, platelet activation indices like MPV and PDW should be altered than the controls. The aim of this study was to evaluate the alterations of platelet indices and hemoglobin level and to investigate whether they can be used as a predictor marker for threatened preterm birth or not.This study was a case control study included 60 pregnant women attending to the department which was divided into two groups: Study group (30 women): with threatened preterm labor and Control group (30 women): healthy pregnant wome Hemoglobin levels were measured and Platelet indices: mean platelet volume (MPV); platelet distribution width (PDW). Lower hemoglobin levels in TPL group. Also, MPV was lower in TPL group while PDW was higher in PTL group. hemoglobin level less than $9.07 \mathrm{~g} / \mathrm{dl}$ may predict TPL also, MPV levels may be used for discriminating high risk population for preterm labor. By using a cut-off value 8.59 for MPV with sensitivity and specificity of 64\%, 46\% respectively PDW in this study revealed an area under curve (AUC) $63 \%$. By using a cut-off value 15.98 for PDW, sensitivity was $68 \%$ and specificity was $47 \%$ for TPL. Conclusion: Preliminary findings of this study showed that hemoglobin level less than $9.07 \mathrm{~g} / \mathrm{dl}$ may predict TPL also, MPV levels may be used for discriminating high risk population for preterm labor. Further prospective studies with larger sample size are needed to confirm this finding.
\end{abstract}

\section{Introduction}

The association between maternal anemia and preterm delivery is controversial. Several studies have showed that maternal anemia was associated with preterm labor [1], whereas some studies found no correlation between maternal anemia and poor pregnancy outcomes including preterm delivery [2].

Complete blood count (CBC) is routinely checked for all pregnant women. Platelet count and platelet indices such as MPV and PDW are parameters of the routinely checked CBC. Being natural sources of growth factors like insulin-like growth factor 1 (IGF1), platelet-derived growth factor (PDGF), vascular endothelial growth factor (VEGF), or transforming growth factor $\beta$ (TGF- $\beta$ ) caused the platelets to have important role in different processes such as inflammation, angiogenesis, repair and regeneration of the tissues [3].

In Threatened PTL patients, due to inflammation and possible ongoing ischemia process, platelet activation indices like MPV and PDW should be altered than the controls. Previous studies suggested that in inflammation via some cytokines, the platelets size and volume alter differently: in low grade inflammatory disorders, by the involvement of the large platelets in thrombi, MPV values may increase [4].

On the other hand, in high grade inflammatory conditions, the consumption of the large platelets at the inflammation site cause a decrease in MPV levels. It is well known that pregnancy itself and also labor causes platelet activation. Platelet indices vary also depending on the gestational week. In general, dilutional thrombocytopenia exists with an compensatory increase in MPV and PDW levels during the pregnancy [4]. Cardiovascular risk factors like smoking status, hypertension, dyslipidemia, diabetes also affect the size of platelets [5].
The aim of this study was to evaluate the alterations of platelet indices and hemoglobin level and to investigate whether they can be used as a predictor marker for threatened preterm birth or not.

\section{Patient and Method}

This study was a case control study that was done in Benha University hospital, obstetrics and gynecology department \& kafr El Sheikh General hospital. The study included 60 pregnant women attending to the department which was divided into two groups: labor

Study group (30 women): with threatened preterm Control group (30 women): healthy pregnant women.

Both groups were pregnant in early third trimester between 29-30 weeks.

\section{Inclusion criteria}

- Patient aged from 20 to 30 years.

- Gestational age from 29-30 weeks.

\section{Criteria of PTL}

- History of previous preterm labor in the study group.

- Contractions occurring at a frequency of four in 20 minutes or eight in 60 minutes plus progressive change in the cervix

- Cervical dilatation greater than $1 \mathrm{~cm}$.

- Cervical effacement of $80 \%$ or greater

- But Threatened preterm labor may be diagnosed when there is documented uterine contraction but no evidence of cervical change 


\section{Exclusion criteria}

Patients with premature preterm rupture of membranes (PPROM) with or without proven or suspected maternal or fetal infection.

Patients with chronic inflammatory diseases, i.e. connective tissue disorders such as systemic lupus erythematosus, rheumatoid arthritis, renal or hepatic insufficiency, hemoglobinopathies, diabetes mellitus, hypertensive disorders, previous myocardial infarction, previous thrombosis history.

All patients in the study were admitted to the hospital were subjected to full history taking, General examination, Regional examination, Abdominal Examination, Deep palpation, Ultrasound examination and the followingLaboratory investigation:

- Routine laboratory investigation; CBC, Rh, coagulation profile, random blood sugar, urine analysis, renal and liver function tests.

- Specific investigations;

- Hemoglobin level.

- Platelet indices: mean platelet volume (MPV); platelet distribution width (PDW).

\section{Results}

The mean age of TPL group was $25.67 \pm 1.29$ and the mean age of the control group was $25.97 \pm 0.96$ ( $p$ > 0.313 ). The mean gestational week of TPL group was $30.97 \pm 0.89$ and the mean gestational week of the control group was $30.87 \pm 0.73(\mathrm{p}=0.636)$.

The main demographic data about gravidity, parity, abortion, gestational week and maternal age showed insignificant statistical differences between both groups.

The mean gestational week of TPL group was $30.97 \pm 0.89$ and the mean gestational week of the control group was $30.87 \pm 0.73(\mathrm{p}=0.636)$.

This table shows highly statistically significant difference between both groups as regard to hemoglobin, hematocrit, WBC, MPV and PDW.
The mean hemoglobin levels were $9.07 \pm 0.51 \mathrm{~g} / \mathrm{dl}$ and $11.26 \pm 0.42 \mathrm{~g} / \mathrm{dl}$ in TPL and control group, respectively $(p<0.001)$. The mean hemoglobin levels were significantly lower in the study group.

MPV levels were $8.59 \pm 0.26(\mathrm{fL})$ and $9.61 \pm 0.19$ (fL) in TPL and control group, respectively $(\mathrm{p}<0.001)$. MPV levels were significantly lower in the study group.

PDW levels were $15.98 \pm 0.33$ and $14.22 \pm 0.41)$ in TPL and control group, respectively $(\mathrm{p}<0.001)$. PDW levels were significantly higher in the study group.

The mean MPV and hemoglobin levels were significantly lower in TPL group and PDW levels were significantly higher.

Regarding the platelet count, there was no statistically significant difference between the TPL and control groups $(\mathrm{p}$ value $=0.521)$.

TPL occurrence had significant strong indirect correlation with hemoglobin and MPV ( $\mathrm{r}=-0.867$, $0.856 \& \mathrm{p}<0.001$, respectively), while had significant strong direct correlation with PDW $(\mathrm{r}=0.866$, $\mathrm{p}<0.001$ ).

In present study AUROC (area under ROC) was 0.621 for hemoglobin which concluded that it is fair predictor to assess TPL. Optimum cut-off was determined using the Youden index was less than 9.07 $\mathrm{g} / \mathrm{dl}$. Sensitivity, specificity was calculated $67 \%, 48 \%$ respectively.

In present study AUROC (area under ROC) was 0.609 for MPV which concluded that it is fair predictor to assess TPL. Optimum cut-off was determined using the Youden index was less than $8.59 \mathrm{~g} / \mathrm{dl}$. Sensitivity, specificity was calculated $64 \%, 46 \%$ respectively.

In present study AUROC (area under ROC) was 0.63 for PDW which concluded that it is fair predictor to assess TPL. Optimum cut-off was determined using the Youden index was less than $15.98 \mathrm{~g} / \mathrm{dl}$. Sensitivity, specificity was calculated $68 \%, 47 \%$ respectively.

Table (1) Peripheral blood changes of the studied groups.

\begin{tabular}{|c|c|c|c|c|}
\hline & \multicolumn{2}{|c|}{ Groups } & \multirow[t]{2}{*}{ t-value } & \multirow[t]{2}{*}{ P-value } \\
\hline & $\underset{\substack{(\mathrm{n}=30) \\
\text { TPL group }}}{ }$ & $\begin{array}{c}\text { Control }_{\operatorname{group}_{(\mathrm{n}=30)}}\end{array}$ & & \\
\hline Hemoglobin $^{(\mathrm{g} / \mathrm{dl})}($ mean \pm SD $)$ & $9.07 \pm 0.51$ & $11.26 \pm 0.42$ & 31.25 & $<0.001^{1} *$ \\
\hline Hematocrit $^{(\mathrm{g} / \mathrm{dl})}($ mean \pm SD $)$ & $28.17 \pm 1.2$ & $34.17 \pm 0.83$ & 22.42 & $<0.001^{1 *}$ \\
\hline $\mathrm{WBCs}^{(/ \mathrm{mm} 3)}(\operatorname{mean} \pm \mathrm{SD})$ & $3953 \pm 584$ & $7458 \pm 2636$ & 7.11 & $<0.001^{1} *$ \\
\hline Platelet count ${ }^{(/ \mathrm{mm} 3)}($ mean $+\mathrm{SD})$ & $214 \pm 16.75$ & $219 \pm 12.41$ & 1.23 & $0.521^{1}$ \\
\hline MPV $^{(f 1)}(\operatorname{mean} \pm$ SD $)$ & $8.59 \pm 0.26$ & $9.61 \pm 0.19$ & 17.36 & $<0.001^{1 *}$ \\
\hline PDW $($ mean \pm SD $)$ & $15.98 \pm 0.33$ & $14.22 \pm 0.41$ & 18.44 & $<0.001^{1} *$ \\
\hline
\end{tabular}

Independent t-test; *Statistically significant when $\mathrm{p}$-value $<0.05$.

Abbreviations: $\quad$ WBCs=white blood cells; $\mathrm{MPV}=$ mean platelet volume; $\mathrm{PDW}=$ platelet distribution width
This table shows highly statistically significant difference between both groups as regard to hemoglobin, hematocrit, WBC, MPV and PDW. 
The mean hemoglobin levels were9.07 $\pm 0.51 \mathrm{~g} / \mathrm{dl}$ and $11.26 \pm 0.42 \mathrm{~g} / \mathrm{dl}$ in TPL and control group, respectively $(\mathrm{p}<0.001)$. The mean hemoglobin levels were significantly lower in the study group.

MPV levels were $8.59 \pm 0.26(\mathrm{fL})$ and $9.61 \pm 0.19$ (fL) in TPL and control group, respectively $(p<0.001)$. MPV levels were significantly lower in the study group.
PDW levels were 15.98 \pm 0.33 and $14.22 \pm 0.41)$ in TPL and control group, respectively $(\mathrm{p}<0.001)$. PDW levels were significantly higher in the study group.

The mean MPV and hemoglobin levels were significantly lower in TPL group and PDW levels were significantly higher.

Regarding the platelet count, there was no statistically significant difference between the TPL and control groups $(\mathrm{p}$ value $=0.521)$.

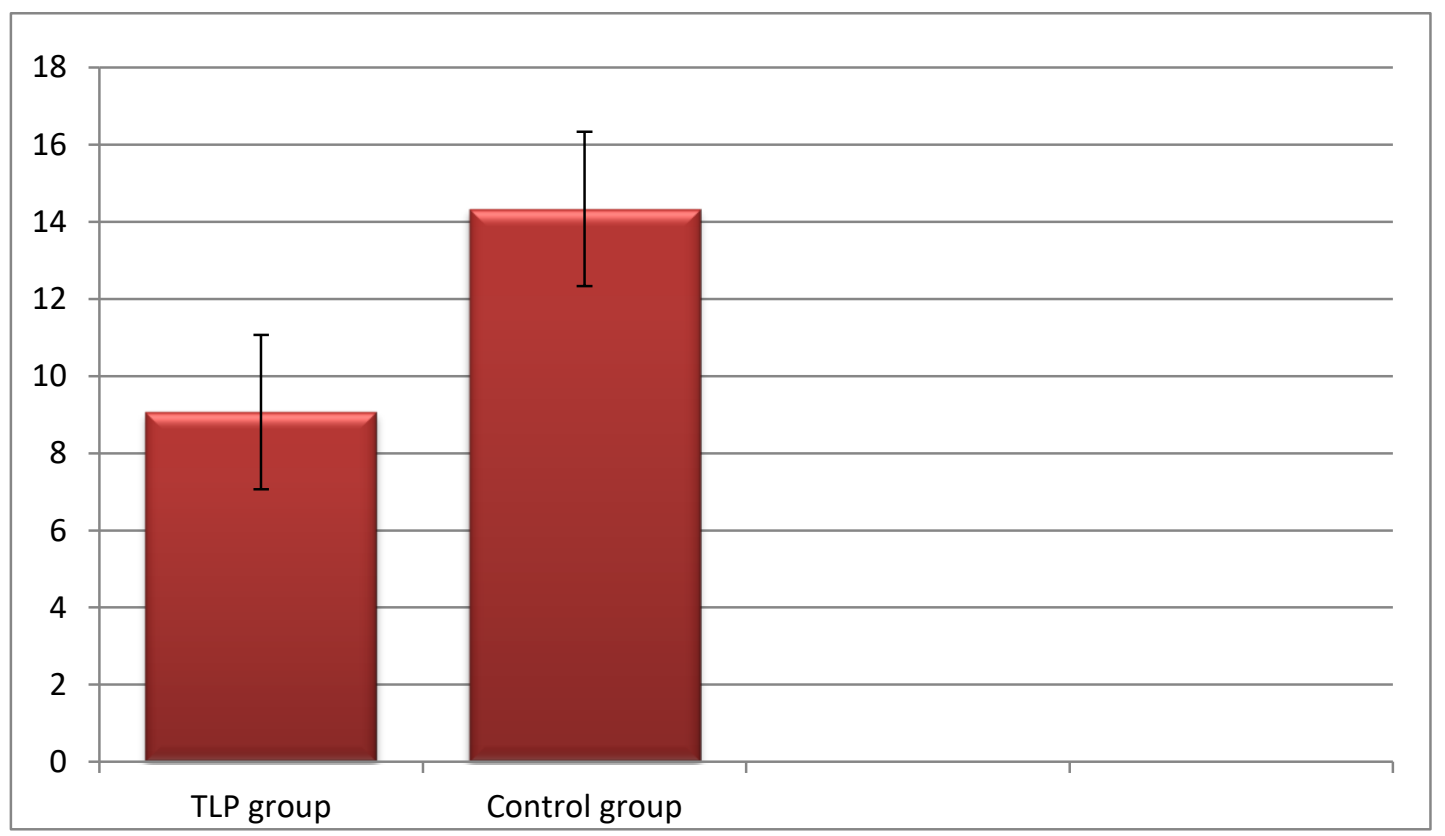

Fig (1) Mean hemoglobin of both groups.

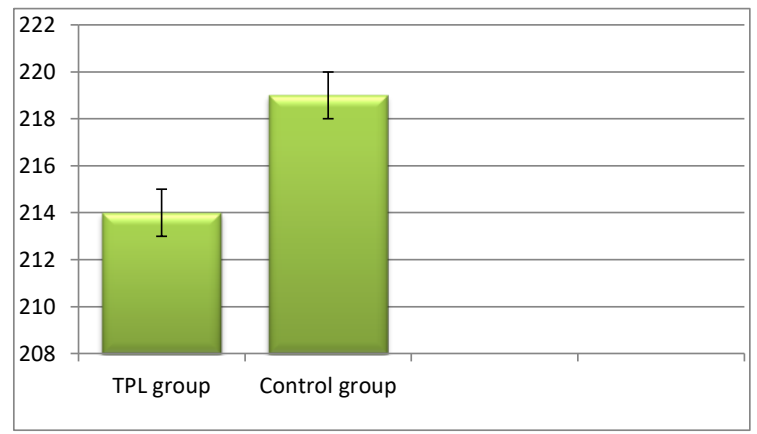

Fig (2) Mean platelet count of both groups. 


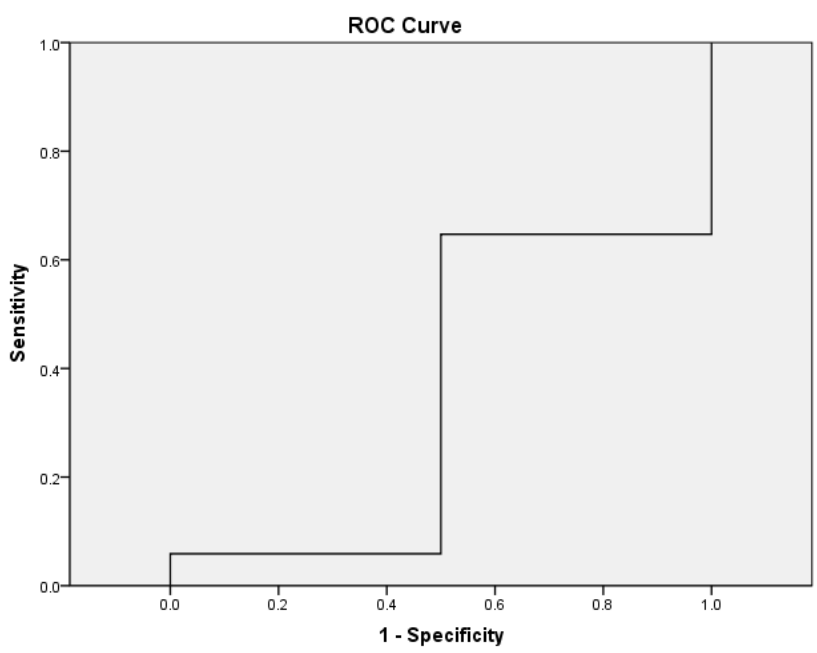

Fig (3) ROC curve of Hemoglobin.

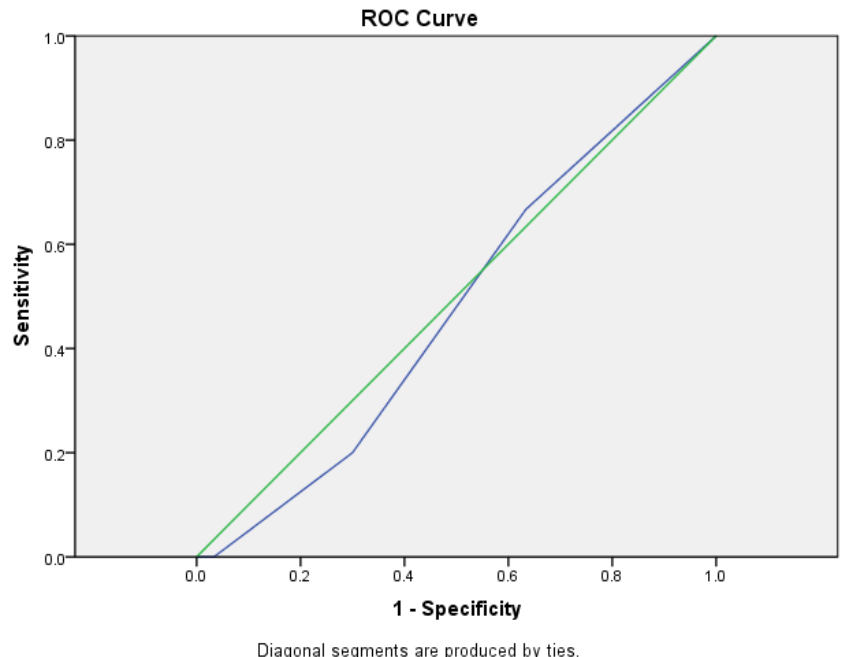

Fig (4) ROC curve of MPV.

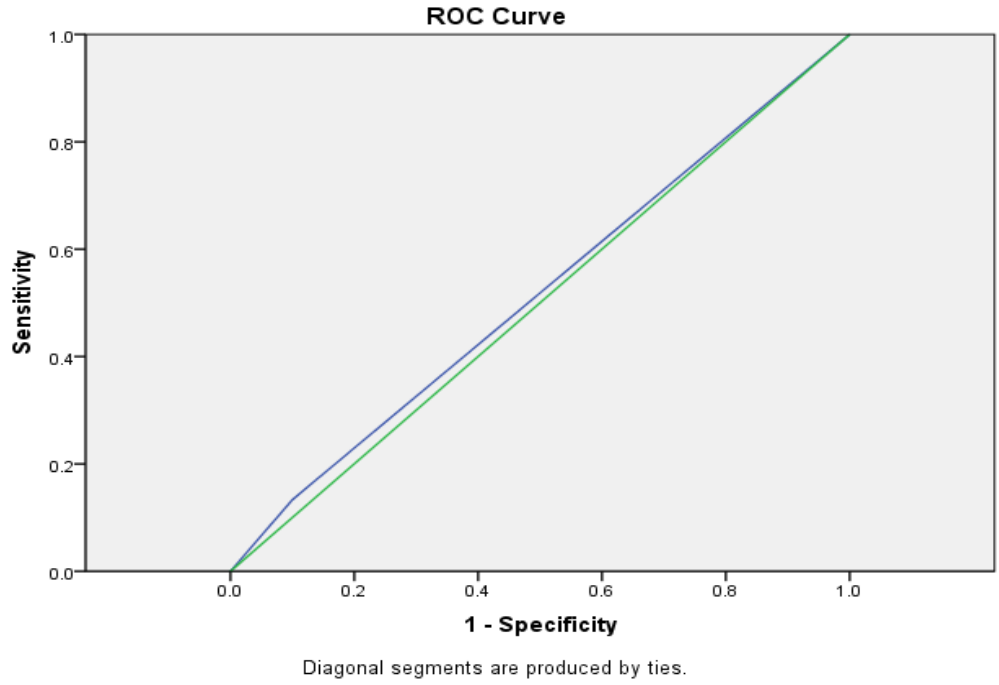

Fig (5) ROC curve of PDW. 


\section{Discussion}

Platelet indices vary also depending on the gestational week. In general, dilutional thrombocytopenia exists with a compensatory increase in MPV and PDW levels during the pregnancy [6,7].

In this study, the mean hemoglobin levels were $9.07 \pm 0.5 \mathrm{~g} / \mathrm{dl}$ and $14.33 \pm 0.67 \mathrm{~g} / \mathrm{dl}$ in TPL and control group, respectively $(\mathrm{p}<0.001)$. The mean hemoglobin levels were significantly lower in TPL group. The association between maternal anemia and preterm delivery is controversial. Several studies have showed that maternal anemia was associated with preterm labor [1], whereas some studies found no correlation between maternal anemia and poor pregnancy outcomes including preterm delivery [5,2].

As a result, preliminary findings of this study showed that hemoglobin level less than $9.07 \mathrm{~g} / \mathrm{dl}$ may predict TPL with sensitivity, specificity were calculated $67 \%, 48 \%$ respectively.

This is similar to Artunc Ulkumen et al., [8] study about " Platelet distribution width (PDW): A putative marker for threatened preterm labour" that included 201 pregnant women with threatened preterm labour admitted between 2009 and 2013 and 192 healthy pregnancies was conducted and found that the mean hemoglobin levels were $9.8 \pm 3.8 \mathrm{~g} / \mathrm{dl}$ and $11.26 \pm 0.42 \mathrm{~g} /$ $\mathrm{dl}$ in TPL and control group, respectively $(\mathrm{p}=0.01)$ also The mean hemoglobin levels were significantly lower in TPL group.

In this study patients of TLP group had mean hematocrit levels significantly lower $28.17 \pm 1.2$ than control group $34.17 \pm 0.83 \quad(\mathrm{p}<0.001)$. This result agree with the study of Artunc Ulkumen et al., [8] who found that the mean hematocrit levels were $29.1 \pm 10.3 \mathrm{~g} / \mathrm{dl}$ and $33.5 \pm 9.5 \mathrm{~g} / \mathrm{dl}$ in TPL and control group, respectively $(\mathrm{p}=0.005)$ also mean hematocrit levels were significantly lower in TPL group.

The mean platelet count was $214 \pm 16.75 / \mathrm{mm} 3$ and $239 \pm 12.41 / \mathrm{mm} 3$, in study and control groups with insignificant difference $(\mathrm{p}=0.521)$.

Also, Artunc Ulkumen et al., [8] found that mean platelet count had no statistically significant difference between the TPL and control groups.

In the current study, MPV levels were $8.59 \pm 0.26$ and 9.61 \pm 0.19 , in TPL and in control groups, respectively $(\mathrm{p}<0.001)$. Where lower MPV levels in this study may be the result of the possible high-grade inflammation in the etiology of TPL.

Also, about PDW levels were $15.98 \pm 0.33$ and $14.22 \pm 0.41)$ in TPL and control group, respectively $(p<0.001)$. PDW levels were significantly higher in the study group.This is similar to Artunc Ulkumen et al., [8] who found that MPV levels were significantly lower in TPL group $(\mathrm{p}=0.01)$; whereas PDW levels were significantly higher in TPL group $(p=0.05)$.

All blood samples in this study were collected in tubes containing potassium ethylenediaminetetraacetate (EDTA) as the anticoagulant agent and they were analyzed in two hours following the sampling. Previous studies mentioned some difficulties in interpreting the results about the platelet indices. The fact that MPV and PDW levels change in a timedependent manner lead to some different results according to the time period between the blood sampling and sample analyzing. Furthermore,

the use of different anticoagulant agents in the blood tube also affect the platelet indices [9]. These above-mentioned factors may be the cause of different comments in previous studies about platelet indices $[10,11]$.

However, previous studies showed that MPV values are not affected if the measurement is within one hour of blood sampling [12].

This is in agreement with Artunc Ulkumen et al., [8] study found that MPV levels were $9.23 \pm 1.38$ and $9.71 \pm 1.47$, PDW levels were $16.78 \pm 0.96$ and $15.75 \pm 10.25$ in TPL and in control groups, respectively $(\mathrm{p}=0.01,0.05$, respectively).

ROC curve analysis for MPV levels of this study patients revealed that MPV levels may be used for discriminating high risk population for preterm labour. By using a cut-off value 8.59 for MPV with sensitivity and specificity of $64 \%, 46 \%$ respectively.

Also, ROC curve analysis for PDW in this study revealed an area under curve (AUC) 63\%. By using a cut-off value 15.98 for PDW, sensitivity was $68 \%$ and specificity was $47 \%$ for TPL.

This is in agreement with Artunc Ulkumen et al., [8] study who found that by using a cut-off value 16.15 for PDW, sensitivity was $76.1 \%$ and specificity was $43.5 \%$ for TPL.

In this study it is noticed that MPV levels were significantly lower and PDW levels were significantly higher in the TPL group, which created a hope for us that decreasing MPV and increasing PDW levels may predict TPL.

\section{References}

[1] A.Levy, D.Fraser, M.Katz. Maternal anemia during pregnancy is an independent risk factor for low birthweight and preterm delivery. Eur J Obstet Gynecol Reprod Biol, Vol. 122, PP.182-186, 2005.

[2] Z.M.Lu, R.L.Goldenberg, S.P.Cliver. The relationship between maternal hematocrit and pregnancy outcome. Obstet Gynecol,Vol.71, PP.190-194,1991.

[3] A.Turgut, M.E.Sak, A.Ozler. Alterations of peripheral blood cells in tubal ectopic pregnancy. Ginekologica Polsaka J., Vol. 84, PP.193-196, 2013.

[4] Q.Zhang, C.V.Ananth, Z.Li. Maternal anaemia and preterm birth: A prospective cohort study. International Journal of Epidemiology,Vol. 38(5), PP.1380-1389,2009.

[5] T.T.Lao, T.C.Pun. Anaemia in pregnancy - is the current definition meaningful? Eur J Obstet Gynecol Reprod Biol,Vol. 68, PP.53-58, 1996.

[6] O.Dundar, P.Yoruk, L.Tutuncu. Longitudinal study of platelet size changes in gestation and predictive 
power of elevated MPV in development of preeclampsia. Prenat Diag, Vol. 28, PP.1052-1056, 2008.

[7] C.Beyan, K.Kaptan, A.Ifran. Platelet count, mean platelet volume, platelet distribution width, and plateletcrit do not correlate with optical platelet aggegation responses in healthy volunteers. .Journal of Thrombosis and Thrombolysis, Vol.22(3), PP.161-164, 2006.

[8] B.Artunc Ulkumen, H.G.Pala, E.Calik. Platelet distribution width (PDW): A putative marker for threatened preterm labour. Pak J Med Sci (PAKISTAN JOURNAL OF MEDICAL SCIENCES),Vol.30(4), PP.745-748, 2014.

[9] E.Vagdatli, E.Gounari, E.Lazaridou. Platelet distribution width: A simple, practical and specific marker of activation of coagulation. Hippokratia journal.Vol. 14, PP.28-32, 2010.

[10] A.Y.Gasparyen, L.Ayvazyen, D.Mikhalidis. MPV: A link between thrombosis and inflammation?Current Pharmaceutical Design.Vol.17(1), PP.47-58,2011.

[11] M.R.Holthe, A.C.Staff, L.N.Berge. Different levels of platelet activation in preeclamptic, normotensive pregnant, and nonpregnant women. Am J Obstet Gynecol.Vol. 190,PP.1128$1134,2004$.

[12] A.Ihara, T.Kawamoto, K.Matsumoto, S.Shouno. Relationship between hemostatic factors and the platelet index in patients with ischemic heart disease. Pathophysiology of Haemostasis and Thrombosis.Vol.35(5), PP.388-391,2006. 